\title{
Photon-Photon Collisions and Photon Structure Functions at LEP
}

\author{
J. Patt ${ }^{\mathrm{a}}$
}

${ }^{a}$ Fakultät für Physik, Albert-Ludwigs-Universität Freiburg, Germany

The present knowledge of the structure of the photon based on measurements of photon structure functions is discussed. This review covers recent results on QED structure functions and on the hadronic structure function $F_{2}^{\gamma}$.

\section{INTRODUCTION}

One of the most powerful tools to investigate the structure of quasi-real photons is the measurement of photon structure functions in deep inelastic electron-photon scattering. At $\mathrm{e}^{+} \mathrm{e}^{-}$colliders, one of the beam electrons radiates a highly virtual photon, described by its negative four-momentum squared $Q^{2}$, that interacts with a quasi-real photon $\left(P^{2} \approx 0\right)$ radiated off the second beam electron probing the structure of the quasi-real photon. This process can be regarded as the scattering of an electron off a quasi-real photon. The deep inelastic electron-photon scattering cross-section may be expressed in terms of structure functions as

$\frac{d^{2} \sigma_{\mathrm{e} \gamma \rightarrow \mathrm{eX}}}{d x d Q^{2}}=\frac{2 \pi \alpha^{2}}{x Q^{4}}\left[\left(1+(1-y)^{2}\right) F_{2}^{\gamma}\left(x, Q^{2}\right)-y^{2} F_{\mathrm{L}}^{\gamma}\left(x, Q^{2}\right)\right]$.

By measuring the deep inelastic electron-photon scattering cross-section the photon structure function $F_{2}^{\gamma}$ can be obtained which is proportional to the parton content of the photon and therefore reveals the structure of the photon. The variables $x$ and $y$ are the usual dimensionless variables of deep inelastic scattering and $\alpha$ is the fine structure constant. The event selection ensures that $y$ is very small $(y \ll 1)$, so the contribution of the term proportional to $F_{\mathrm{L}}^{\gamma}\left(x, Q^{2}\right)$ is usually neglected. The negative four-momentum squared $Q^{2}$ is determined with good precision by measuring the energy and the scattering angle of the electron radiating the highly virtual photon. For the determination of $x$ the measurement of the invariant mass $W$ of the photon-photon system is necessary. The invariant mass $W$ and thus $x$ can be accurately measured in case of $\mu^{+} \mu^{-}$final states, studied for the determination of QED structure functions. But for hadronic final states the measurement of $W$ is a source of significant uncertainties since the measured invariant mass of an event is usually smaller than the true one. Thus all LEP experiments use unfolding methods to relate the measured $x$ distribution to the true one for the determination of the hadronic structure function $F_{2}^{\gamma}$.

Measurements of photon structure functions have by now a tradition of almost 20 years since the first $F_{2}^{\gamma}$ was obtained by PLUTO [1]. The LEP accelerator is a unique place for the measurements of photon structure functions because of the large accessible $Q^{2}$ range owing to the various beam energies covered at LEP. 


\section{THE QED STRUCTURE FUNCTIONS}

Leptonic final states, especially the $\mu^{+} \mu^{-}$final state, provide a very clean environment to study the leptonic structure function $F_{2, \text { QED }}^{\gamma}$ of the photon. $F_{2, \mathrm{QED}}^{\gamma}$ parametrizes the crosssection of the pure QED process. The invariant mass of the $\mu^{+} \mu^{-}$final state $W$ and thus $x$ is well known allowing a precise determination of $F_{2, \mathrm{QED}}^{\gamma}$. Measurements of $F_{2 \text {,QED }}^{\gamma}$ have been performed by all four LEP collaborations [2-6]. Fig. 1 shows the world summary of the $F_{2, \text { QED }}^{\gamma}$ measurements ranging from $\left\langle Q^{2}\right\rangle$ below $1 \mathrm{GeV}^{2}$ up to $130 \mathrm{GeV}^{2}$ (see Ref. [7] and references therein). $F_{2, \text { QED }}^{\gamma}$ rises with increasing $x$ up to values of close to one. All measurements are well described by the QED predictions. The LEP data are even precise enough to study the effect of the small virtuality of the quasi-real photon and it has been demonstrated that the QED calculations assuming $P^{2} \equiv 0$ yield a too large $F_{2, \mathrm{QED}}^{\gamma}$ compared to the data.

By measuring the azimuthal angle $\chi$ between the plane containing the muon pair and the plane containing the incoming quasi-real photon and the deep inelastic scattered electron measurements of the structure functions $F_{\mathrm{A}, \mathrm{QED}}^{\gamma}$ and $F_{\mathrm{B}, \mathrm{QED}}^{\gamma}$ are possible. $F_{\mathrm{A}, \mathrm{QED}}^{\gamma}$ and $F_{\mathrm{B}, \mathrm{QED}}^{\gamma}$ enter in a more differential parametrisation of
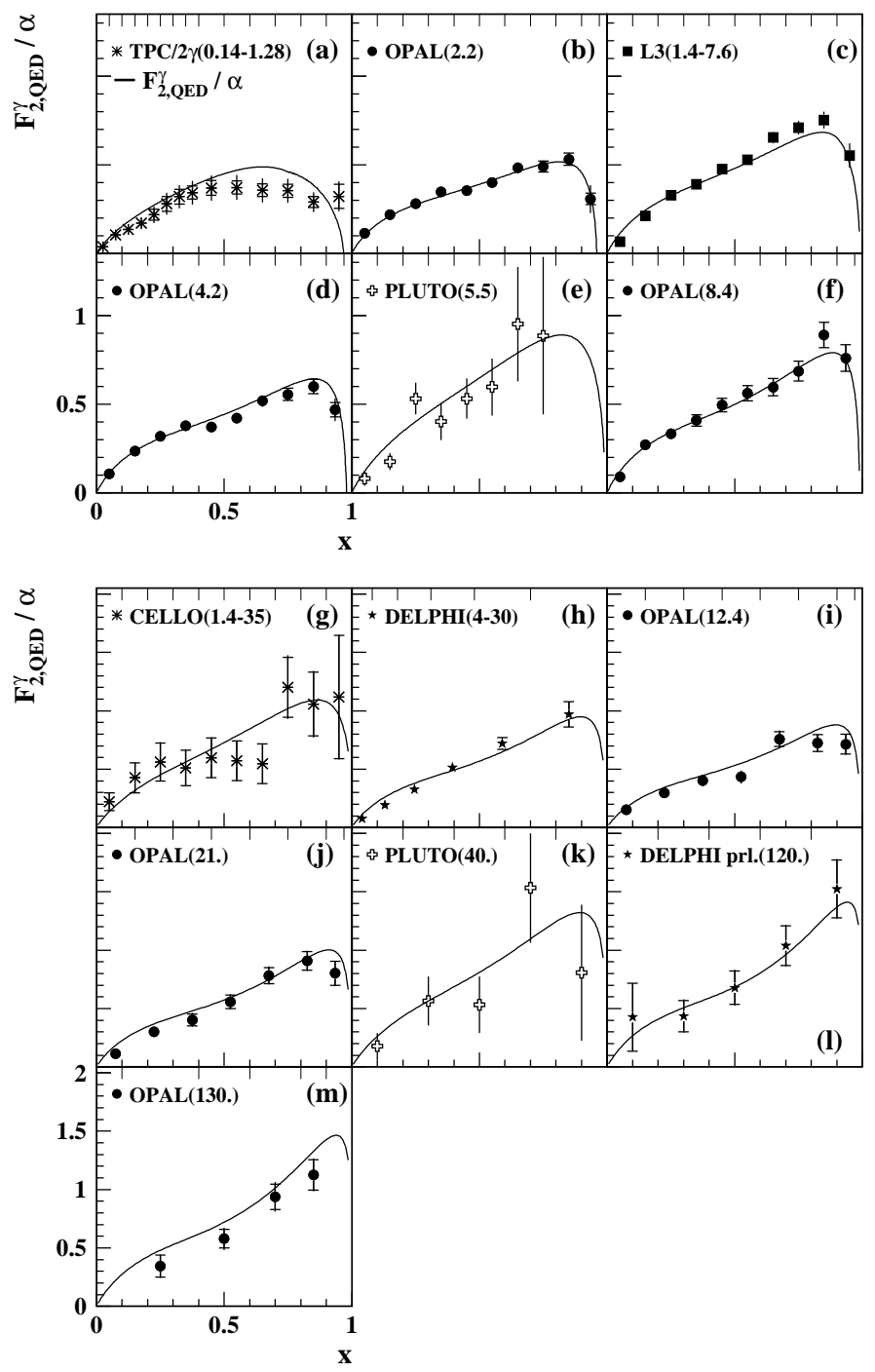

Figure 1: The measurements of $F_{2, \mathrm{QED}}^{\gamma}$ as a function of $x$ for various $\left\langle Q^{2}\right\rangle$ compared to $Q E D$. The quoted errors for h) are statistical only. The Figure is taken from Ref. [7].

the deep inelastic electron-photon scattering cross-section and are not accessible in the cross-section measurement. Azimuthal correlations based on measurements of the $\chi$ distribution were also investigated by all four LEP collaborations $[2-4,6]$.

\section{THE HADRONIC STRUCTURE FUNCTION $\boldsymbol{F}_{2}^{\gamma}$}

The measurement of the hadronic structure function $F_{2}^{\gamma}$ has attracted a lot of interest at LEP over the last years. In contrast to $F_{2, \mathrm{QED}}^{\gamma}, F_{2}^{\gamma}$ describes the pointlike as well as the hadron-like component of the photon structure. Two distinct features of the photon structure are of special interest. On the one hand the shape of $F_{2}^{\gamma}$ is measured 
as a function of $x$ at fixed values of $Q^{2}$. Particular emphasis is put on measuring the low- $x$ behaviour of $F_{2}^{\gamma}$ since a rise towards small $x$ is expected due to the hadron-like component of the photon similar as in the case of the proton structure function measured at HERA. On the other hand, the evolution of $F_{2}^{\gamma}$ with $Q^{2}$ at medium $x$ is investigated that is predicted by QCD to show a logarithmic rise.

The LEP collaborations have measured $F_{2}^{\gamma}$ in the range $1.86<\left\langle Q^{2}\right\rangle<400 \mathrm{GeV}^{2}$ and $x>0.002 \quad[8,5,9-11]$. Fig. 2 shows the world data of $F_{2}^{\gamma}$ as a function of $x$ in different bins of $\left\langle Q^{2}\right\rangle$ (see Ref. [7] and references therein). $\quad F_{2}^{\gamma}$ is found to rise smoothly towards large $x$ as $F_{2, \text { QED }}^{\gamma}$, but the predicted rise towards low $x$ which has been investigated by OPAL and L3 at small values of $\left\langle Q^{2}\right\rangle$ can not be established yet (Fig. $2 \mathrm{a}$ and b). Due to improved Monte Carlo programs the systematic errors due to model dependent uncertainties are not the dominant errors any more. The total systematic errors are now of comparable size to the statistical errors. All measurements are satisfactorily described by several of the existing $F_{2}^{\gamma}$ parametrisations, e.g. GRV(HO) [12] and SaS [13].

The evolution of $F_{2}^{\gamma}$ with $Q^{2}$ has been studied for different bins in $x$ profiting of the large accessible $Q^{2}$ range. A summary of the $F_{2}^{\gamma}$ measurements as a function of $Q^{2}$ at medium $x$ is shown in Fig 3. Unfortunately, the different experiments quote their results for different ranges in $x$. This makes a comparison more difficult, because the predictions for different ranges in $x$ start to be significantly different for $Q^{2}>100 \mathrm{GeV}^{2}$, as can be seen in Fig. 3 . The measurements are consistent with each other and a clear rise of $F_{2}^{\gamma}$ with $Q^{2}$ is observed.
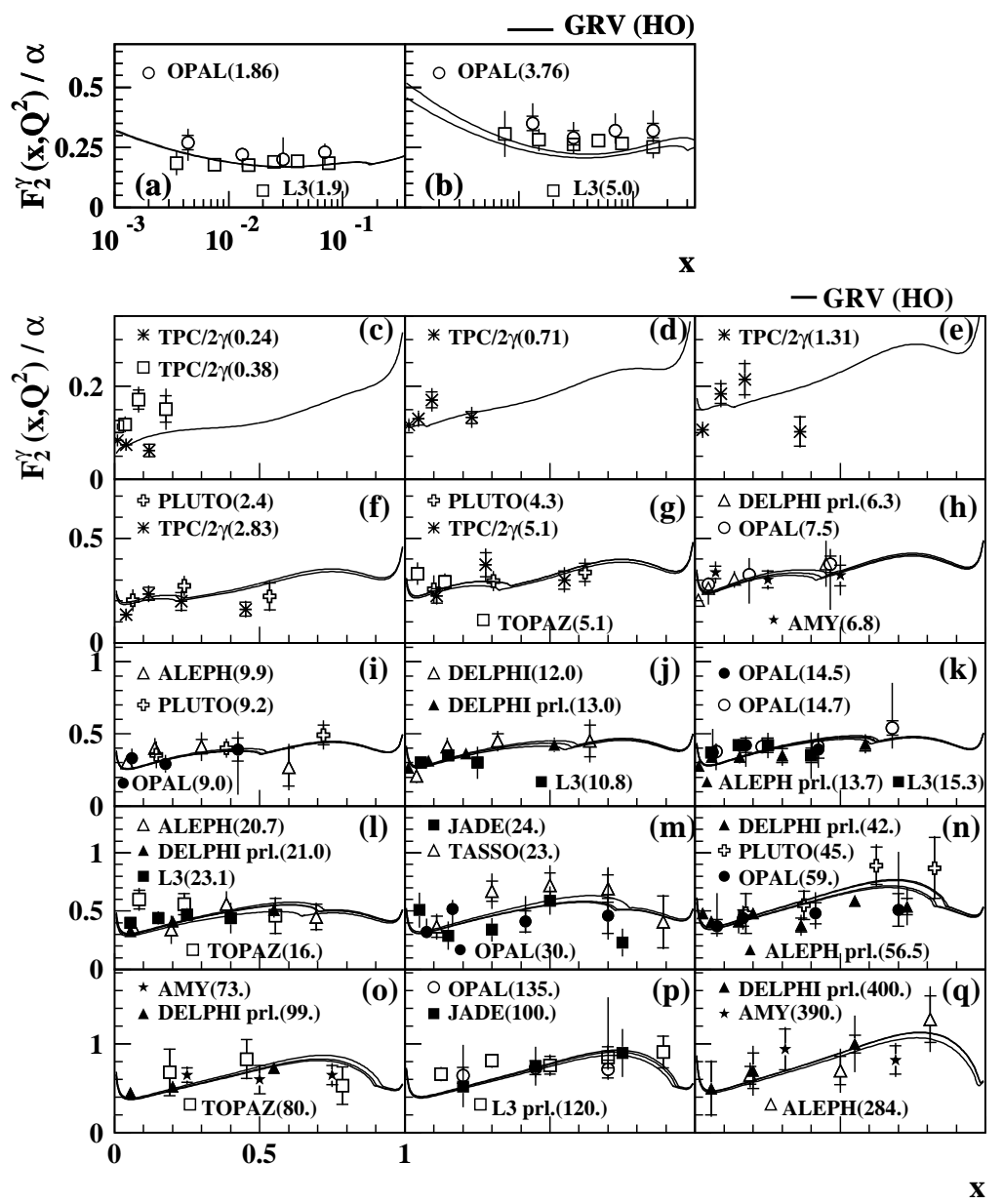

Figure 2: The world data on $F_{2}^{\gamma}$ as a function of $x$ unfolded on a logarithmic scale, a) and b), or on a linear scale, c)-q), compared to the prediction of the GRV(HO) parametrisation. The inner error bar is the statistical error and the outer the quadratic sum of the statistical and systematic error. The Figure is taken from Ref. [7]. 


\section{CONCLUSION}

Informations on the photon structure in a large range in $Q^{2}$ and $x$ have been obtained at LEP based on measurements of photon structure functions. The QED predictions of the structure of the photon are found to be in good agreement with the experimental results. Concerning the hadronlike structure of the photon, the logarithmic rise of $F_{2}^{\gamma}$ with increasing $Q^{2}$ is clearly seen, but the predicted rise towards small $x$ can not be established yet.

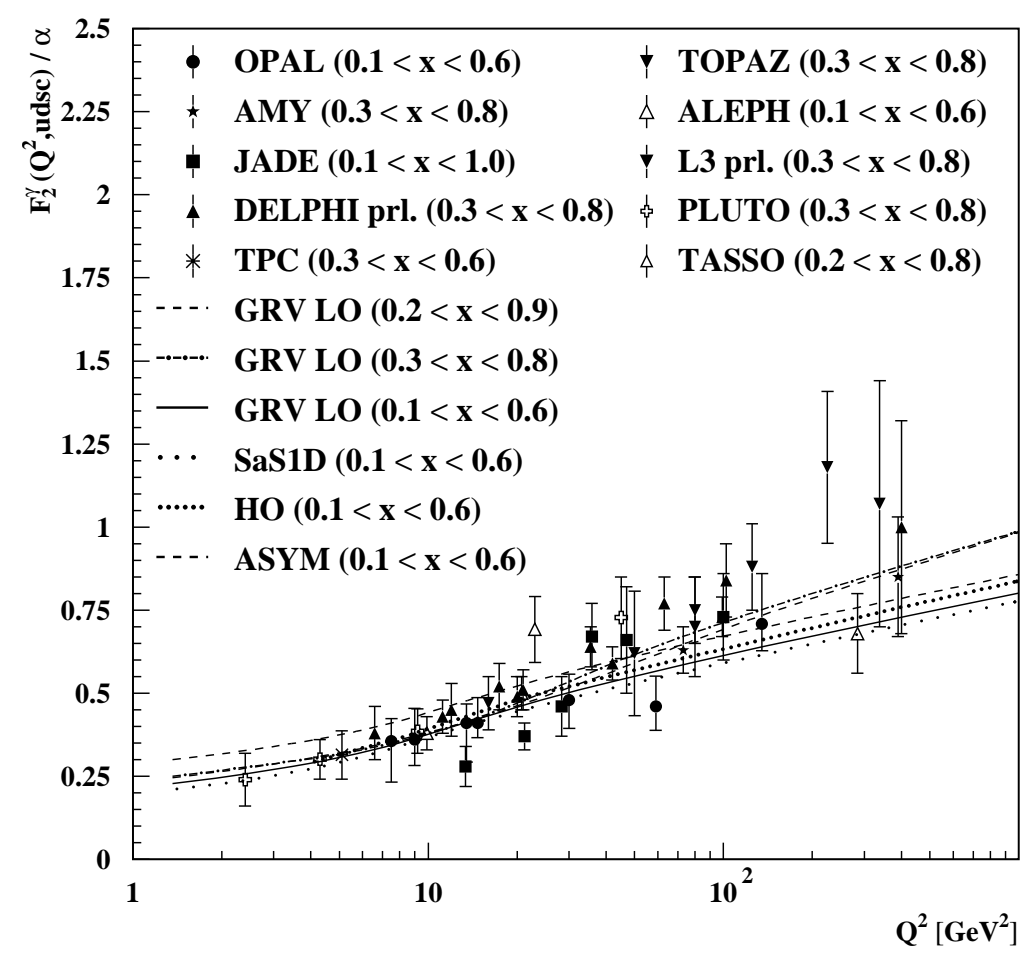

Figure 3: Summary of the measurements of $F_{2}^{\gamma}$ as a function of $Q^{2}$ at medium $x$ compared to several theoretical predictions taking into account various ranges in $x$. The quoted errors are the quadratic sum of the statistical and systematic error. The Figure is taken from Ref. [7].

\section{REFERENCES}

1. PLUTO Coll., C. Berger et al., Phys. Lett. B107 (1981) 168.

2. OPAL Coll., G. Abbiendi et al., CERN-EP/99-010, accepted by Euro. Phys. J. C (1999).

3. L3 Coll., M. Acciarri et al., Phys. Lett. B438 (1998) 363.

4. ALEPH Coll., C. Brew, proceedings of PHOTON97, Egmond aan Zee, 1997.

5. DELPHI Coll., P. Abreu et al., Z. Phys. C69 (1996) 223.

6. DELPHI Coll., A. Zintchenko, proceedings of PHOTON99, Freiburg, 1999.

7. R. Nisius, The Structure of Quasi-Real and Virtual Photons, hep-ex/9907012.

8. OPAL Coll., K. Ackerstaff et al., Z. Phys. C74 (1997) 33; K. Ackerstaff et al., Phys. Lett. B411 (1997) 387; K. Ackerstaff et al., Phys. Lett. B412 (1997) 225.

9. DELPHI Coll., I. Tyapkin, proceedings of PHOTON97, Egmond aan Zee, 1997; I. Tyapkin, proceedings of 'Workshop on photon interactions and the photon structure', Lund, 1998.

10. L3 Coll., M. Acciarri et al., Phys. Lett. B436 (1998) 436; M. Acciarri et al., Phys. Lett. B447 (1999) 147; F.C. Erné, proceedings of PHOTON99, Freiburg, 1999.

11. ALEPH Coll., R. Barate et al., Phys. Lett. B458 (1999) 152; A. Böhrer, proceedings of PHOTON99, Freiburg, 1999.

12. M. Glück, E. Reya and A. Vogt, Phys. Rev. D46 (1992) 1973; M. Glück, E. Reya and A. Vogt, Phys. Rev. D45 (1992) 3986.

13. G.A. Schuler and T. Sjöstrand, Z. Phys. C68 (1995) 607. 